\title{
Time and Frequency Synchronization for OFDMA Uplink System using the SAGE Algorithm
}

\author{
Jong-Ho Lee and Seong-Cheol Kim, Member, IEEE
}

\begin{abstract}
In this letter, we propose an iterative time and frequency synchronization scheme for orthogonal frequencydivision multiple access (OFDMA) uplink system using the space-alternating generalized expectation-maximization (SAGE) algorithm. In the proposed scheme, the expectation step of the SAGE algorithm is intended to remove the multiple-access interference (MAI) caused by the other asynchronous users. Then, the maximization step is utilized in the timing and frequency offset estimation of each asynchronous user using the MAI-canceled signals. Simulation results present that the proposed scheme shows performance enhancement in the presence of multiple asynchronous users.
\end{abstract}

Index Terms-Synchronization, multiple access interference (MAI), orthogonal frequency division multiple access (OFDMA), space-alternating generalized expectation-maximization (SAGE).

\section{INTRODUCTION}

$\mathbf{O}$ RTHOGONAL frequency-division multiple access (OFDMA) is a multiplexing technique where each user employs a different set of orthogonal sub-carriers to transmit data simultaneously. The different orthogonal sub-carrier sets for different users in OFDMA system make it possible to avoid multiple access interference (MAI). Therefore, it has become an attractive solution for various wireless communication systems [1]-[4].

It is known that time and frequency synchronization is critical for OFDMA uplink system. With imperfect synchronization, inter-carrier interference (ICI) and inter-symbol interference (ISI) are generated due to the loss of sub-carrier orthogonality, which induce both co-channel and inter-channel interference. Moreover, carrier frequency synchronization for the uplink of an OFDMA system is more difficult, since the frequency recovery for one user may result in the misalignment of the other synchronized users.

Carrier frequency synchronization techniques for singleuser OFDM system have been studied in several papers [5]-[10]. In [5] and [6], the frequency synchronization is achieved using the redundancy generated by the cyclic prefix. The synchronization schemes proposed in [7]-[9] employ the maximum length sequence in the frequency-domain. In [10], the pseudo-noise (PN) based preamble in the time-domain is used to achieve the carrier frequency synchronization. Based

Manuscript received June 5, 2005; revised September 12, 2005 and December 20, 2005; accepted February 14, 2006. The associate editor coordinating the review of this paper and approving it for publication was R. Fantacci. This work was supported by the University IT Research Center Project and Brain Korea 21 Project.

The authors are with the Institute of New Media and Communications, School of Electrical Engineering and Computer Science, Seoul National University, Seoul, 151-742, Korea (email: \{dowar, sckim\}@maxwell.snu.ac.kr).

Digital Object Identifier 10.1109/TWC.2007.05390. on these results, [1] and [3] proposed timing and frequency synchronization schemes for the uplink of an OFDMA system. Then, through the downlink control channel, the estimated offsets in the base station (BS) are returned to each user's terminal where timing and frequency offsets are actually adjusted.

In [1], the available spectrum is divided into several subbands by grouping adjacent sub-carriers. Then, each user is allocated in one of the sub-bands and the BS obtains the user-separated signal in the time-domain through sub-band filters. However, in the sub-band based OFDMA uplink, deep fading due to the frequency selective characteristic may lie in several adjacent sub-carriers so that full channel diversity is not guaranteed. In order to utilize the channel diversity for all users, the interleaved sub-carrier set can be assigned to each user. Then, the user-separated signals are available only in the frequency-domain and [3] proposed a time and frequency synchronization scheme for the interleaved OFDMA uplink to estimate the timing and frequency offsets of a new user entering the system, assuming that the other active users are perfectly synchronized to the BS. Moreover, [2] suggested a precoding technique for OFDMA systems to enhance the bandwidth and power efficiency. In [4], the carrier frequency offset correction scheme is proposed to consider the lease square (LS) and minimum mean square error (MMSE) criteria, assuming that frequency synchronization errors are known by the BS.

In this letter, we consider the situation, where multiple asynchronous users are present in the interleaved OFDMA uplink system. Then, the synchronization scheme in [3] should be operated in the presence of MAI induced by the other asynchronous users. In order to overcome the MAI, we extend the results of [3] and propose an iterative method to estimate the timing and frequency offsets of each asynchronous user via the space-alternating generalized expectationmaximization (SAGE) algorithm [11].

The SAGE algorithm has been usually employed in the study of the direction of arrival (DOA) estimation [12], multiuser detection and channel estimation [13], [14] and multiuser acquisition for code division multiple access (CDMA) systems [15]. For the interleaved OFDMA uplink, [16] proposed a joint frequency offset and channel estimation scheme using the SAGE algorithm. This scheme, however, assumes that timing synchronization is not required. Moreover, it deals with the time-domain received signal, which is the superposition of all active users' signals. In order to initiate the iteration in [16], the other users' signals are assumed as noise to make use of the frequency offset estimator proposed for singleuser system or the initial estimate of frequency offset is set 
to be zero. Then, the rough initial estimate brings a large number of iterations, which results in the significant increase of computational complexity.

In the proposed scheme, we deal with the user-separated signals in the frequency-domain, which may include the MAI due to timing and frequency offsets. Here, the expectation step of the proposed scheme is utilized to remove the MAI in the received signal of each asynchronous user. Then, the maximization step is intended to estimate timing and frequency offsets.

\section{System Model}

Let $N$ be the number of sub-carriers and $K_{T}$ be the total number of users in the system. The number of sub-carriers assigned to each user can be given as $P=N / K_{T}$ and the transmitted symbol vector for the $(k)$ th user at the $(l)$ th OFDM block is defined by $\mathbf{s}_{k, l}=\left[s_{k, l}(0), s_{k, l}(1), \cdots, s_{k, l}\left(P_{-}\right.\right.$ 1)] $]^{T}$ where $1 \leq k \leq K_{T}$. Then, the time-domain transmitted signal for the $(k)$ th user at the $(l)$ th OFDM block can be expressed as

$$
b_{k, l}(n)=\frac{1}{\sqrt{N}} \sum_{p=0}^{P-1} s_{k, l}(p) \exp \left(j \frac{2 \pi c_{k}(p) n}{N}\right), 0 \leq n<N
$$

where $c_{k}(p)$ denotes the $(p)$ th sub-carrier index assigned to the ( $k$ )th user. Note that the insertion of $N_{G}$-point cyclic prefix yields $b_{k, l}(n)=b_{k, l}(n+N)$ where $-N_{G} \leq n<0$ and the total number of samples including the cyclic prefix is given by $N_{T}=N+N_{G}$.

Here, let $K_{A}$ be the number of asynchronous users trying to access the given system. Without loss of generality, we assume that the user $\# 1$ to user $\# K_{A}$ are asynchronous and the user $\#\left(K_{A}+1\right)$ to user $\# K_{T}$ are ideally aligned. Here, perfect synchronization is not possible to achieve in practice and we assume that the MAI induced by aligned users is almost negligible. Then, the time-domain received signal at the $(l)$ th block can be given in the matrix form shown as

$$
\mathbf{y}_{l}=\sum_{k=1}^{K_{A}} e^{j \frac{2 \pi v_{k} l N_{T}}{N}} \boldsymbol{\Gamma}\left(v_{k}\right) \mathbf{D}_{l}\left(\mu_{k}\right) \mathbf{h}_{k}+\sum_{k=K_{A}+1}^{K_{T}} \mathbf{B}_{k, l} \mathbf{h}_{k}+\mathbf{w}_{l}
$$

where $v_{k}$ and $\mu_{k}$ denote the frequency and timing offsets for the $(k)$ th asynchronous user, respectively. Moreover, $\mathbf{h}_{k}=$ $\left[h_{k}(0), h_{k}(1), \cdots, h_{k}(J-1)\right]^{T}$ is the channel impulse response (CIR) vector for the $(k)$ th user with $J$ multipaths. In [3], it is shown that timing offset due to the propagation delay for each user is decomposed in an integer part and a fractional part. The integer part becomes $\mu_{k}$ and the fractional part is included in the sampled CIR $\mathbf{h}_{k}$. In (2), $\boldsymbol{\Gamma}\left(v_{k}\right)$ is a diagonal matrix defined as

$$
\boldsymbol{\Gamma}\left(v_{k}\right)=\operatorname{diag}\left\{\exp \left(j \frac{2 \pi v_{k} n}{N}\right), 0 \leq n<N\right\} .
$$

$\mathbf{B}_{k, l}$ and $\mathbf{D}_{l}\left(\mu_{k}\right)$ in (2) are given as $\left[\mathbf{B}_{k, l}\right]_{n, j}=b_{k, l}(n-j)$ and

$$
\left[\mathbf{D}_{l}\left(\mu_{k}\right)\right]_{n, j}=\left\{\begin{array}{c}
b_{k, l}\left(n-j-\mu_{k}\right) \\
-N_{G}+\mu_{k} \leq n-j<N \\
b_{k, l-1}\left(n-j-\mu_{k}+N_{T}\right), \\
-N_{G} \leq n-j<-N_{G}+\mu_{k}
\end{array}\right.
$$

where $0 \leq n<N$ and $0 \leq j<J$. The additive white Gaussian noise (AWGN) vector $\mathbf{w}_{l}$ has the covariance matrix $\sigma^{2} \mathbf{I}_{N}$ where $\mathbf{I}_{N}$ is a $N \times N$ identity matrix.

Then, the frequency-domain received signal vector for the $(m)$ th asynchronous user $\left(1 \leq m \leq K_{A}\right)$ can be obtained by

$$
\begin{aligned}
\mathbf{x}_{m, l}= & \mathbf{F}_{m} \mathbf{y}_{l} \\
= & \boldsymbol{\Phi}_{m, l}\left(v_{m}, \mu_{m}, \mathbf{h}_{m}\right) \\
& +\sum_{\substack{k=1 \\
k \neq m}}^{K_{A}} \boldsymbol{\Phi}_{m, l}\left(v_{k}, \mu_{k}, \mathbf{h}_{k}\right)+\mathbf{v}_{m, l}
\end{aligned}
$$

where

$$
\begin{array}{r}
\boldsymbol{\Phi}_{m, l}\left(v_{k}, \mu_{k}, \mathbf{h}_{k}\right)=e^{j \frac{2 \pi v_{k} l N_{T}}{N}} \mathbf{F}_{m} \boldsymbol{\Gamma}\left(v_{k}\right) \mathbf{D}_{l}\left(\mu_{k}\right) \mathbf{h}_{k}, \\
\mathbf{F}_{m}=\frac{1}{\sqrt{N}} \exp \left(-j \frac{2 \pi c_{m}(p) n}{N}\right), 0 \leq p<P
\end{array}
$$

and $\mathbf{v}_{m, l}=\mathbf{F}_{m} \mathbf{w}_{l}$. Note that $\mathbf{F}_{m} \mathbf{B}_{k, l}=\mathbf{0}_{P \times J}$ where $\mathbf{0}_{P \times J}$ denotes a $P \times J$ matrix with all zero entries and $\left(K_{A}+1\right) \leq k \leq K_{T}$. It is seen that the first term in the right-hand side of $(5)$ denotes the desired signal of the $(m)$ th asynchronous user and the second term in (5) presents the disturbance signal induced by the other asynchronous users. The proposed scheme in this letter is motivated to overcome these MAI signals.

\section{TIME AND FREQUENCY SYNCHRONIZATION VIA THE SAGE ALGORITHM}

The proposed iterative scheme employs the SAGE algorithm, which is composed of the expectation and maximization steps.

\section{A. Expectation Step}

In the expectation step of the SAGE algorithm, we intend to divide the received signal of the $(m)$ th asynchronous user $\mathbf{x}_{m, l}$ in (5) into the desired signal

$$
\mathbf{x}_{m, l}^{D}=\boldsymbol{\Phi}_{m, l}\left(v_{m}, \mu_{m}, \mathbf{h}_{m}\right)+\beta_{D} \mathbf{v}_{m, l}
$$

and the MAI signal

$$
\mathbf{x}_{m, l}^{I}=\sum_{\substack{k=1 \\ k \neq m}}^{K_{A}} \boldsymbol{\Phi}_{m, l}\left(v_{k}, \mu_{k}, \mathbf{h}_{k}\right)+\beta_{I} \mathbf{v}_{m, l}
$$

which indicates the transformation from the incomplete observation $\mathbf{x}_{m, l}$ to the complete observations $\mathbf{x}_{m, l}^{D}$ and $\mathbf{x}_{m, l}^{I}$ by the expectation-maximization (EM) algorithm [17], [18]. Then, we try to estimate $v_{m}, \mu_{m}$ and $\mathbf{h}_{m}$ using the desired signal in (8). Here, we assume that each user transmits $(L+1)$ identical OFDM blocks known to the BS. Then, $\mathbf{D}_{l}\left(\mu_{k}\right)$ in (4) and (6) is independent of the OFDM block index $l$, which implies that $\mathbf{D}_{l}\left(\mu_{k}\right)=\mathbf{D}\left(\mu_{k}\right)$.

The expectation step at the $(i)$ th iteration is to evaluate following expectations given as

$$
\begin{aligned}
\left(\mathbf{x}_{m, l}^{D}\right)^{(i)}= & E\left[\mathbf{x}_{m, l}^{D} \mid \hat{\varphi}_{1}^{(i)}, \hat{\varphi}_{2}^{(i)}, \cdots, \hat{\varphi}_{K_{A}}^{(i)}\right] \\
= & \boldsymbol{\Phi}_{m, l}\left(\hat{\varphi}_{m}^{(i)}\right) \\
& +\beta_{D}\left(\mathbf{x}_{m, l}-\sum_{k=1}^{K_{A}} \mathbf{\Phi}_{m, l}\left(\hat{\varphi}_{k}^{(i)}\right)\right),
\end{aligned}
$$




$$
\begin{aligned}
\left(\mathbf{x}_{m, l}^{I}\right)^{(i)}= & E\left[\mathbf{x}_{m, l}^{I} \mid \hat{\varphi}_{1}^{(i)}, \hat{\varphi}_{2}^{(i)}, \cdots, \hat{\varphi}_{K_{A}}^{(i)}\right] \\
= & \sum_{\substack{k=1 \\
k \neq m}}^{K_{A}} \boldsymbol{\Phi}_{m, l}\left(\hat{\varphi}_{k}^{(i)}\right) \\
& +\beta_{I}\left(\mathbf{x}_{m, l}-\sum_{k=1}^{K_{A}} \boldsymbol{\Phi}_{m, l}\left(\hat{\varphi}_{k}^{(i)}\right)\right)
\end{aligned}
$$

where $\hat{\varphi}_{k}^{(i)}$ is the $(i)$ th estimate of $\varphi=\left(v_{k}, \mu_{k}, \mathbf{h}_{k}\right)$, which denotes an unknown parameter vector for the $(k)$ th asynchronous user. Moreover, the nonnegative parameters $\beta_{D}$ and $\beta_{I}$ satisfy $\beta_{D}^{2}+\beta_{I}^{2}=1[12]$.

In (10), it is seen that the expectation step is equivalent to the MAI cancellation process. In the proposed scheme, there is no need to evaluate $\left(\mathbf{x}_{m, l}^{I}\right)^{(i)}$ in (11), because the next maximization step employs only the MAI cancelled signal $\left(\mathbf{x}_{m, l}^{D}\right)^{(i)}$ in (10). Note that the expectation step to evaluate requires $K_{A}^{2} L(N(J+P+1)+2 P)$ times of complex multiplications and $K_{A}^{2} L\left(N(J+P-1)+K_{A} P\right)$ times of complex additions.

\section{B. Maximization Step}

The maximization step at the $(i)$ th iteration sequentially updates the unknown parameters shown as

$$
\begin{gathered}
\hat{v}_{m}^{(i+1)}=\underset{\tilde{v}_{m}}{\operatorname{argmax}} \Lambda\left(\tilde{v}_{m}, \hat{\mu}_{m}^{(i)}, \hat{\mathbf{h}}_{m}^{(i)} \mid\left(\mathbf{x}_{m, l}^{D}\right)^{(i)}\right), \\
\hat{\mu}_{m}^{(i+1)}=\underset{\tilde{\mu}_{m}}{\operatorname{argmax} \Lambda}\left(\hat{v}_{m}^{(i+1)}, \tilde{\mu}_{m}, \hat{\mathbf{h}}_{m}^{(i)} \mid\left(\mathbf{x}_{m, l}^{D}\right)^{(i)}\right), \\
\hat{\mathbf{h}}_{m}^{(i)}=\underset{\tilde{\mathbf{h}}_{m}}{\operatorname{argmax}} \Lambda\left(\hat{v}_{m}^{(i+1)}, \hat{\mu}_{m}^{(i+1)}, \tilde{\mathbf{h}}_{m} \mid\left(\mathbf{x}_{m, l}^{D}\right)^{(i)}\right),
\end{gathered}
$$

where the log-likelihood function $\Lambda\left(\tilde{v}_{m}, \tilde{\mu}_{m}, \tilde{\mathbf{h}}_{m} \mid \mathbf{x}_{m, l}^{D}\right)$ can be given as

$$
\begin{aligned}
& \Lambda\left(\tilde{v}_{m}, \tilde{\mu}_{m}, \tilde{\mathbf{h}}_{m} \mid \mathbf{x}_{m, l}^{D}\right) \\
= & \frac{1}{\left(\pi \sigma^{2}\right)^{2}} \exp \left(-\frac{1}{\sigma^{2}} \sum_{l=1}^{L}\left|\mathbf{x}_{m, l}^{D}-\boldsymbol{\Phi}_{m, l}\left(\tilde{v}_{m}, \tilde{\mu}_{m}, \tilde{\mathbf{h}}_{m}\right)\right|^{2}\right) .
\end{aligned}
$$

In order to extend the results of [3] for the proposed scheme, we define the CIR vector $\tilde{\mathbf{h}}_{m}$ as a function of $\tilde{v}_{m}$ and $\tilde{\mu}_{m}$ as given by the following equation

$$
\tilde{\mathbf{h}}_{m}\left(\tilde{v}_{m}, \tilde{\mu}_{m}\right)=\left[\mathbf{D}^{H}\left(\tilde{\mu}_{m}\right) \mathbf{D}\left(\tilde{\mu}_{m}\right)\right]^{-1} \mathbf{D}^{H}\left(\tilde{\mu}_{m}\right) \mathbf{A}_{m}^{(i)}\left(\tilde{v}_{m}\right)
$$

where

$$
\begin{gathered}
\mathbf{A}_{m}^{(i)}\left(\tilde{v}_{m}\right)=\boldsymbol{\Gamma}^{H}\left(\tilde{v}_{k}\right) \mathbf{F}_{m}^{H} \mathbf{z}_{m}^{(i)} \\
\mathbf{z}_{m}^{(i)}=\frac{1}{L} \sum_{l=1}^{L} \exp \left(-j \frac{2 \pi \tilde{v}_{k} l N_{T}}{N}\right)\left(\mathbf{x}_{m, l}^{D}\right)^{(i)}
\end{gathered}
$$

and the superscript $(.)^{H}$ denotes the conjugated transpose. Then, the frequency offset estimate $\hat{v}_{m}^{(i+1)}$ in (12) can be obtained by the correlation-based frequency offset estimator in [3] using $\left\{\left(\mathbf{x}_{m, l}^{D}\right)^{(i)} ; 1 \leq l \leq L\right\}$. Moreover, substituting

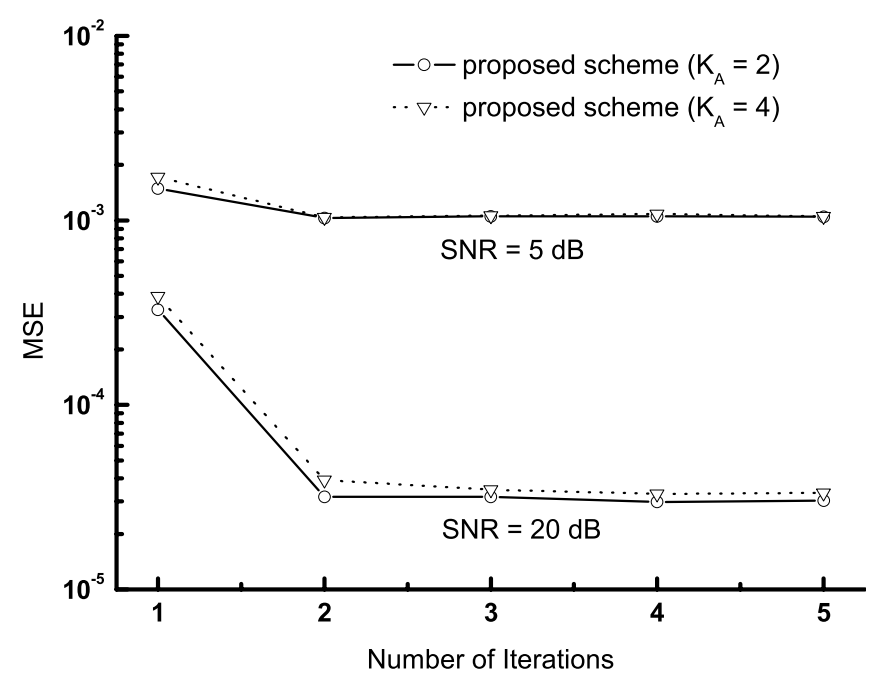

(a)

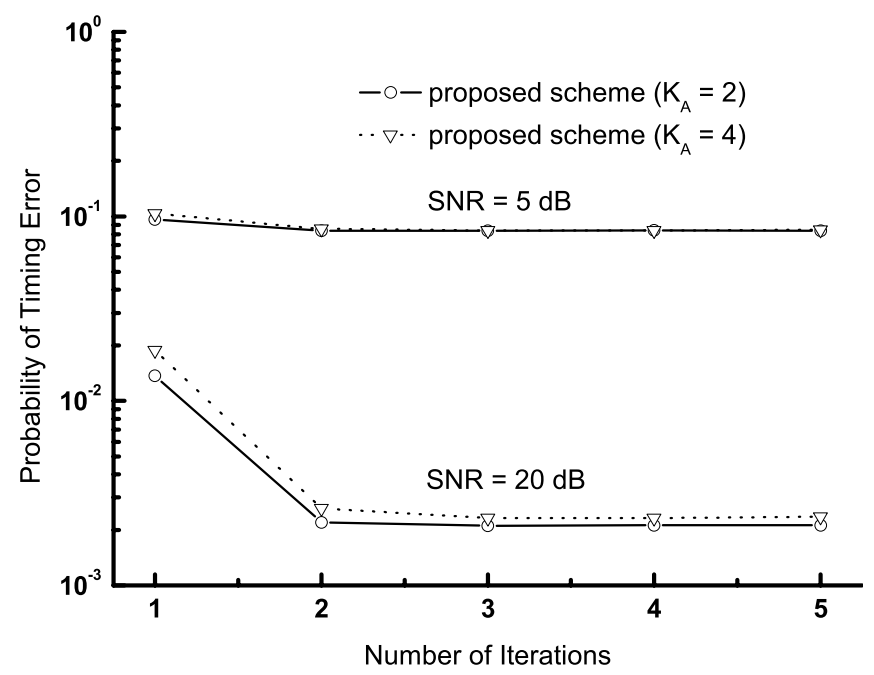

(b)

Fig. 1. Convergence property of proposed scheme. (a) MSE performance for frequency offset estimator, and (b) probability of timing error.

(16) into (15) and keeping (17) in mind, we can evaluate the timing estimate in (13) by a 1-D search shown as

$$
\hat{\mu}_{m}^{(i+1)}=\underset{0 \leq \tilde{\mu}_{m} \leq \mu_{\max }}{\operatorname{argmax}} \mathbf{A}_{m}^{(i)}\left(\hat{v}_{m}^{(i+1)}\right)^{H} \mathbf{V}\left(\tilde{\mu}_{m}\right) \mathbf{A}_{m}^{(i)}\left(\hat{v}_{m}^{(i+1)}\right)
$$

where

$$
\mathbf{V}\left(\tilde{\mu}_{m}\right)=\mathbf{D}\left(\tilde{\mu}_{m}\right)\left[\mathbf{D}^{H}\left(\tilde{\mu}_{m}\right) \mathbf{D}\left(\tilde{\mu}_{m}\right)\right]^{-1} \mathbf{D}^{H}\left(\tilde{\mu}_{m}\right)
$$

and $\mu_{\max }$ denotes the maximum expected value of timing offset. Note that it is impractical to evaluate the matrix inversion in (20) at each iteration. But, it is obvious that $\left\{\mathbf{V}\left(\tilde{\mu}_{m}\right) ; \tilde{\mu}_{m}=0,1, \cdots, \mu_{\max }\right\}$ can be pre-computed and stored in the receiver side, since they do not depend on the frequency offset $v_{m}$ and the CIR vector $\mathbf{h}_{m}$ [3]. Finally, the CIR estimator in (14) given $\hat{v}_{m}^{(i+1)}$ and $\hat{\mu}_{m}^{(i+1)}$ yields $\hat{\mathbf{h}}_{m}^{(i+1)}=$ $\tilde{\mathbf{h}}_{m}\left(\hat{v}_{m}^{(i+1)}, \hat{\mu}_{m}^{(i+1)}\right)$. According to [3], the frequency estimator needs $K_{A} P L(3 L-2) / 8$ times of complex multiplications and $K_{A} P L(3 L-2) / 4$ times of complex additions. Moreover, the timing estimator is involved with $K_{A} P L$ times of complex multiplications, $K_{A} P(P+1)$ times of real multiplications, 


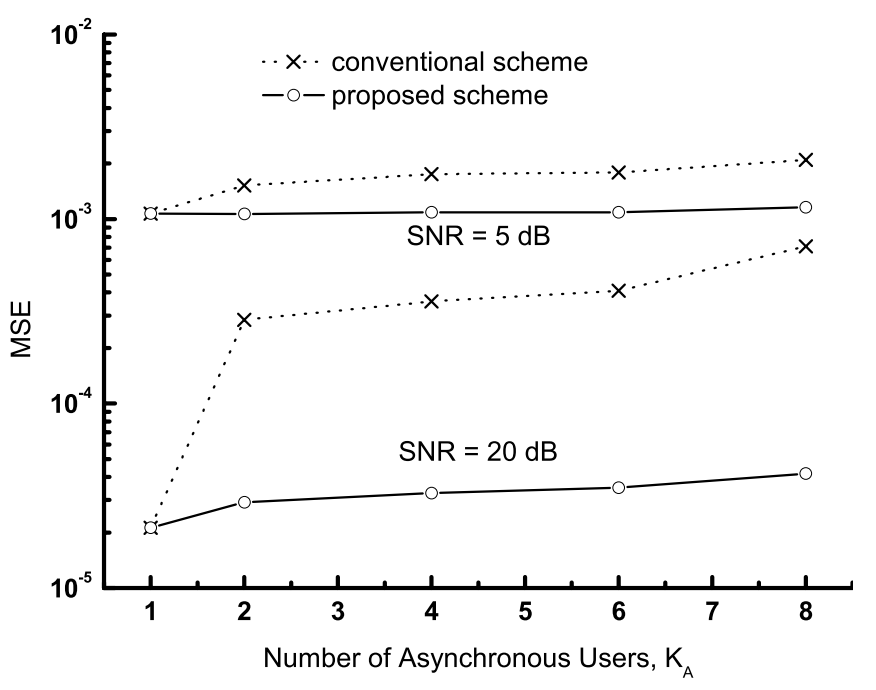

Fig. 2. Comparison of MSE performance for frequency offset estimator according to the number of asynchronous users.

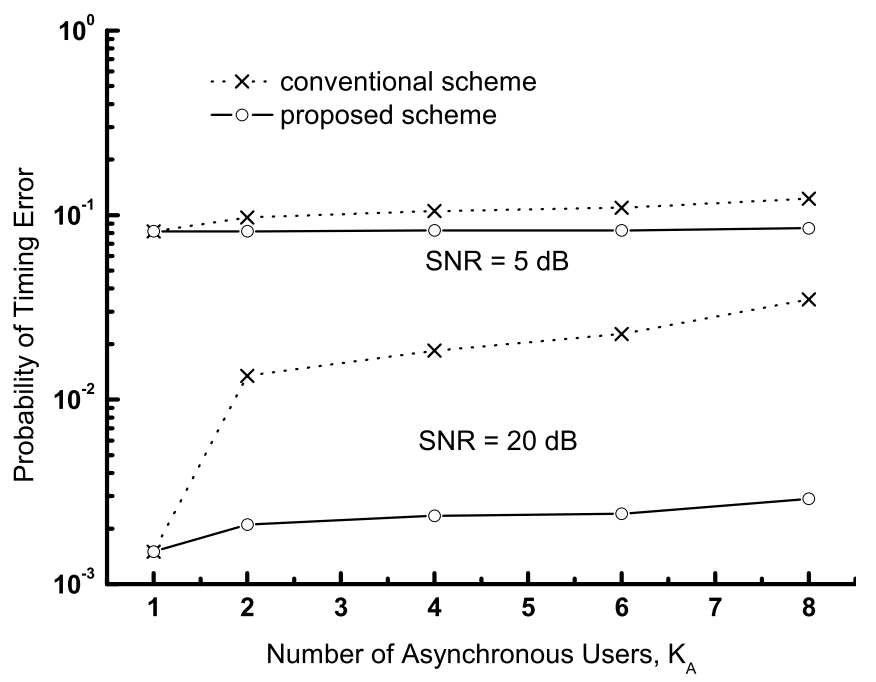

Fig. 3. Comparison of probability of timing error according to the number of asynchronous users.

$K_{A} P(L-1)$ times of complex additions and $K_{A} P(3 P+1) / 2$ times of real additions.

The iteration of the expectation and maximization steps continues until the estimates converge. It is known that $\beta_{D}=$ $\beta_{I}=1$ is the optimal condition to maximize the Fisher information of $\mathbf{x}_{m, l}^{D}$ and $\mathbf{x}_{m, l}^{I}$ in (10) and (11) as given in [11]. Moreover, the initial estimates to begin the iteration of the proposed algorithm are obtained by the synchronization scheme suggested in [3]. Note that the initial estimates may be coarse, since they are evaluated using $\mathbf{x}_{m, l}$ in (55) including the MAI.

\section{Simulation Results}

System parameters in this study are summarized as follows.

- The number of sub-carriers is $N=128$.

- The power delay profile of CIR for each user is given as $E\left[\left|h_{k}(j)\right|^{2}\right] \propto \exp (-j / 4), 0 \leq j<8$ and each multipath varies according to Rayleigh distribution [19].

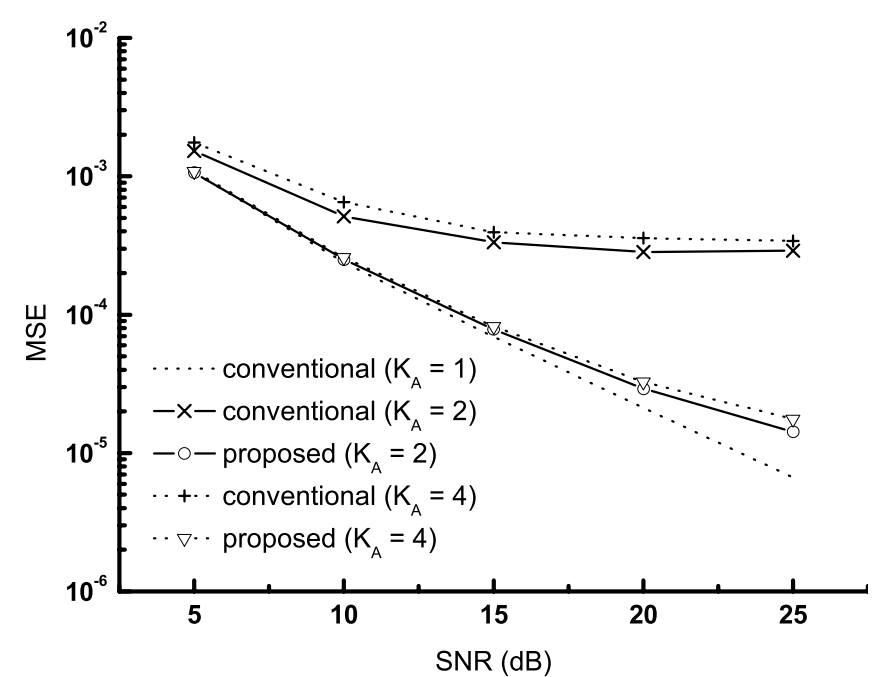

Fig. 4. MSE performance for frequency offset estimator versus SNR.

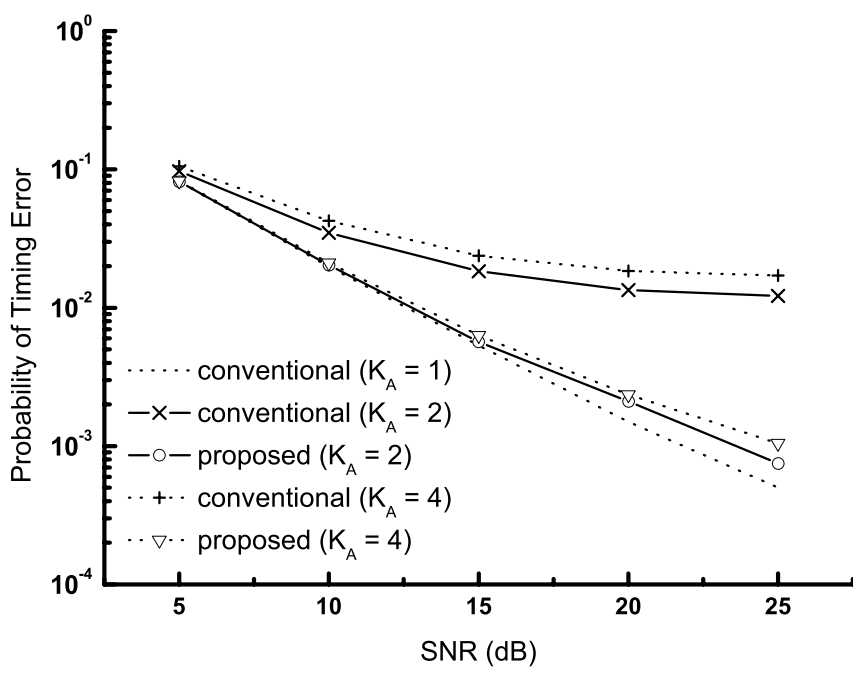

Fig. 5. Probability of timing error versus SNR.

- The CIR of each user is assumed to be static over the $L$ observation blocks.

- The number of samples in the guard interval is set to be $N_{G}=8$.

- The total number of users in the system is $K_{T}=8$.

- Each user employs uniformly spaced sub-carriers from each other, which indicates that the sub-carrier index for the $(k)$ th user is given by $c_{k}(p)=k+(p-1) K T, 1 \leq$ pleqP.

- The sampling period is $0.5 \mu \mathrm{s}$ and the maximum expected value of timing offset $\mu_{m} a x$ is set to be 14 considering the cell radius of $1 \mathrm{~km} \mathrm{[3].}$

- The number of observed OFDM blocks is $L=2$.

- The frequency offset for each asynchronous user is a uniform random variable in the range between -0.4 and 0.4 .

- The timing offset is a uniform random variable in the range between 0 and $\mu_{m} a x$.

In order to provide variable data rates for different users in practice, the number of sub-carriers assigned to the user would not be fixed. As the number of sub-carriers assigned to 
the asynchronous user increases, the MAI to the other users becomes enlarged. In our simulations, however, it is assumed that all users occupy the same number of sub-carriers for simplicity.

Fig. 1 shows that the proposed scheme requires two iterations to obtain the converged mean square error (MSE) performance of frequency offset estimate and the converged probability of timing errors. The signal to noise ratio (SNR) is fixed to be 5 and $20 \mathrm{~dB}$, assuming that the average received power for each user is the same at the BS. Here, the timing errors are defined by the events that the timing estimate induces ISI in the discrete Fourier transform (DFT) output, that is, $\left.\hat{\mu}_{m}^{(} i\right)-\mu_{m}>0$ or $\left.\hat{\mu}_{m}^{(} i\right)-\mu_{m}<J-N_{G}-1$ [3]. Note that the first iteration indicates the initialization process. Here, we fixed the number of iteration to two in the following simulation results.

Fig. 2 presents the MSE performance of the frequency offset estimate according to the number of asynchronous users. It is seen that the conventional frequency offset estimator in [3] shows severely degraded MSE performance as the number of asynchronous user increases, whereas the proposed scheme significantly compensates the performance degradation due to the MAI induced by the other asynchronous users especially at high SNR. Moreover, it is seen that the performance of the proposed scheme is not quite sensitive to the number of asynchronous users, which certifies that the proposed scheme effectively suppresses the disturbance signals due to the other asynchronous users. This is also shown in Fig. 3, which illustrates the probability of timing error.

Fig. 4 presents the MSE performance of frequency offset estimate versus SNR. In the presence of multiple asynchronous users, the conventional scheme shows higher error floors, while the performance of the proposed scheme is quite comparable to that of conventional single user synchronization scheme for both $K_{A}=2$ and 4 in all ranges of SNR. Fig. 5 shows the probability of timing error as a function of SNR. The SNR loss of the proposed scheme against the conventional single user synchronization scheme is within $1 \mathrm{~dB}$ for time and frequency estimates of each asynchronous user. Therefore, it can be concluded that the proposed scheme is suitable for the multiple-user synchronization in the interleaved OFDMA uplink system. However, it is noted that the proposed scheme suffers from the computational load to cancel the MAI in the expectation step at each iteration.

In Fig. 6, we investigate the convergence property of the proposed scheme in the presence of power imbalance. It is assumed that user \#1 suffers the $3 \mathrm{~dB}$ loss in the average received power, while the average power of the other asynchronous users from user $\# 2$ to user $\# K_{A}$ is the same as that of the synchronous users. The SNR in the figure is based on the average received power except for user \#1. As shown in Fig. 6, the convergence property for both timing and frequency offset estimators in the proposed scheme is still maintained regardless of the power loss or the number of asynchronous users.

\section{CONCLUSion}

In this letter, we propose an iterative time and frequency synchronization scheme using the SAGE algorithm for the

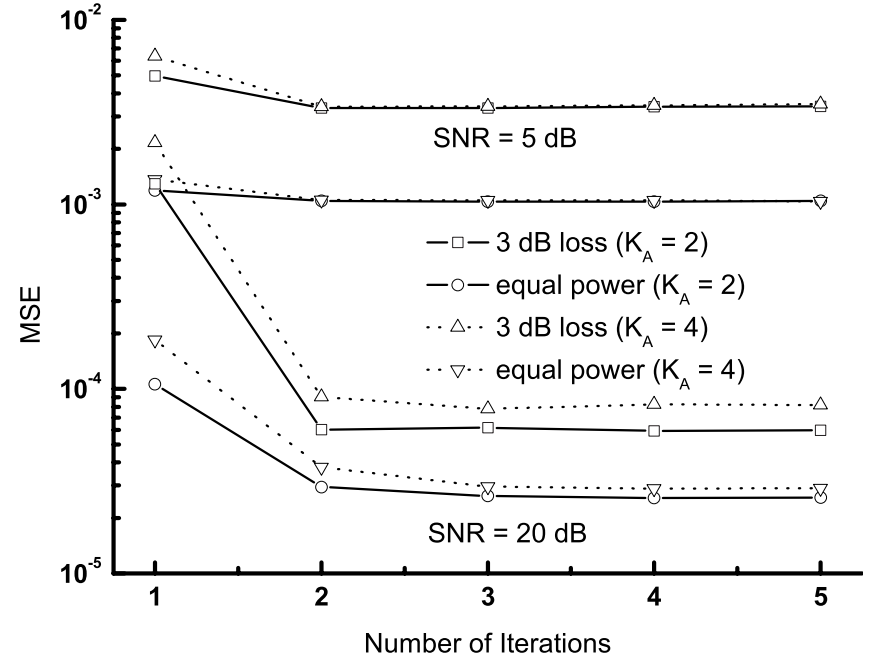

(a)

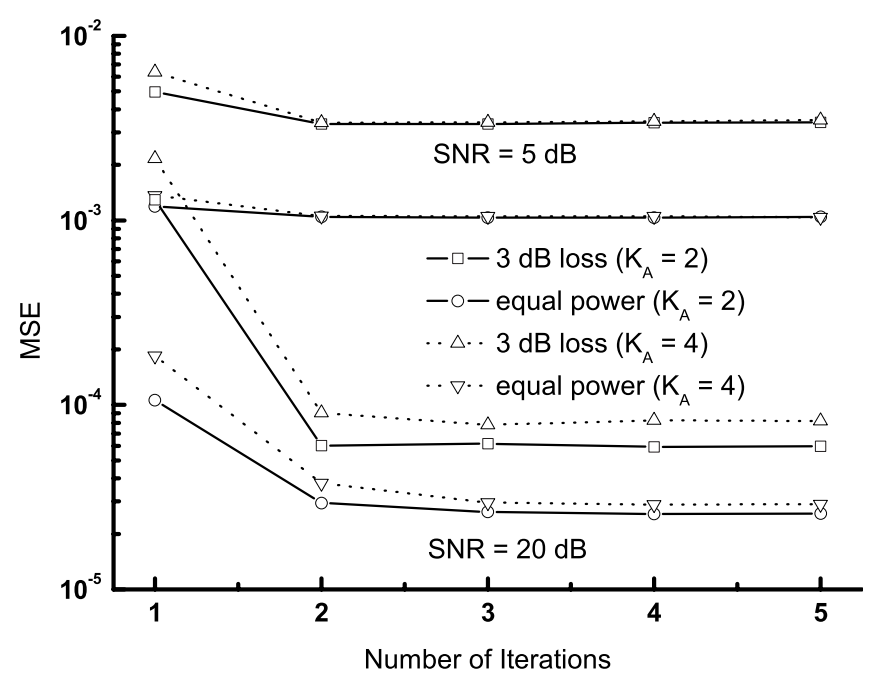

(b)

Fig. 6. Convergence property of proposed scheme in case of power imbalance. (a) MSE performance for frequency offset estimator, and (b) probability of timing error.

interleaved OFDMA uplink system to overcome the MAI in case that multiple asynchronous users are present in the system. The expectation step of the proposed scheme is intended to divide each user's received signal into the desired signal and the MAI signal. Then, the maximization step is utilized to estimate the time and frequency offsets using the desired signals obtained in the expectation step. The proposed scheme requires about two iterations to approach the converged time and frequency offset estimates. Simulation results present that the proposed scheme outperforms the conventional scheme especially at high SNR in the presence of multiple asynchronous users.

\section{ACKNOWLEDGMENT}

The authors would like to thank the paper editor and the reviewers for their valuable comments and suggestions.

\section{REFERENCES}

[1] J. J. van de Beek, P. O. Borjesson, M. L. Boucheret, D. Landstram, J. M. Arenas, P. Odling, C. Ostberg, M. Wahlqvist, and S. K. Wilson, 
"A time and frequency synchronization scheme for muiltiuser OFDM," IEEE J. Sel. Areas Commun., vol. 17, no. 11, pp. 1900-1914, Nov. 1999.

[2] P. Xia, S. Zhou, and G. B. Giannakis, "Bandwith- and power-efficient multicarrier multiple access," IEEE Trans. Commun., vol. 51, no. 11, pp. 1828-1837, Nov. 2003.

[3] M. Morelli, "Timing and frequency synchronization for the uplink of an OFDMA system," IEEE Trans. Commun., vol. 52, no. 2, pp. 296-306, Feb. 2004

[4] Z. Cao, U. Tureli, and Y. D. Yao, "Efficient structure-based carrier frequency offset estimation for interleaved OFDMA uplink," in Proc. International Conference on Communications 2003, pp. 3361-3365.

[5] F. Daffara and O. Adami, "A new frequency detector for orthogonal multicarrier transmission techniques," in Proc. Vehicular Technology Conference 1995, vol. 2, pp. 804-809.

[6] J. J. van de Beek, M. Sandell, and P. O. Borjesson, "ML estimation of timing and frequency offset in OFDM systems," IEEE Trans. Signal Processing, vol. 45, no. 7, pp. 1800-1805, July 1997.

[7] P. H. Moose, "A technique for orthogonal frequency division multiplexing frequency offset correction," IEEE Trans. Commun., vol. 42, no. 10, pp. 2908-2914, Oct. 1994.

[8] T. M. Schmidl and D. C. Cox, "Robust frequency and timing synchronization for OFDM," IEEE Trans. Commun., vol. 45, no. 12, pp. 1613-1621, Dec. 1997.

[9] A. J. Coulson, "Maximum likelihood synchronization for OFDM using a pilot symbol: algorithm," IEEE J. Sel. Areas Commun., vol. 19, no. 12, pp. 2486-2494, Dec. 2001.

[10] F. Tufvesson, O. Edfors, and M. Faulkner, "Time and frequency synchronization for OFDM using PN-sequence preamble," in Proc. Vehicular Technology Conference 1999, vol. 4, pp. 2203-2207.
[11] J. A. Fessler and A. O. Hero, "Space-alternating generalized expectationmaximization algorithm," IEEE Trans. Signal Processing, vol. 42, no. 10, pp. 2664-2677, Oct. 1994.

[12] B. H. Fleury, M. Tschudin, R. Heddergott, D. Dahlhaus, and K. I. Pedersen, "Channel parameter estimation in mobile radio environments using SAGE algorithm," IEEE J. Sel. Areas Commun., vol. 17, no. 3, pp. 434450, Mar. 1999.

[13] H. Dai and H. V. Poor, "Iterative space-time processing for multiuser detection in multipath CDMA channels," IEEE Trans. Signal Processing, vol. 50, no. 9, pp. 2116-2127, Sept. 2002.

[14] A. Kocian and B. H. Fleury, "EM-based joint data detection and channel estimation of DS-CDMA signals," IEEE Trans. Commun., vol. 51, no. 10, pp. 1709-1720, Oct. 2003.

[15] T. Wu, "Iterative multiuser acquisition for DS-CDMA systems," in Proc. Vehicular Technology Conference 2003, vol. 4, pp. 2822-2826.

[16] M. Pun, S. Tsai, and C. Kuo, "An EM-based joint maximum likelihood estimation of carrier frequency offset and channel for uplink OFDMA system," in Proc. Vehicular Technology Conference 2004, vol. 1, pp. 598602.

[17] A. P. Dempster, N. M. Laird, and D. B. Rubin,"Maximum-likelihood from the incomplete data via the EM algorithm," J. Royal Statist. Society, vol. 39, pp. 1-17, 1977.

[18] C. N. Georghiades and J. C. Han, "Sequence estimation in the presence of random parameters via the EM algorithm," IEEE Trans. Commun., vol. 45, no. 3, pp. 300-308, Mar. 1997.

[19] W. C. Y. Lee, Mobile Communications Engineering. New York: McGrow-Hill, 1982. 